\title{
Genetic characterisation and local genotypes of canine parvovirus strains collected from pet dogs in central and eastern China during 2018-2019
}

\author{
Wen $\mathrm{Hu}^{1, *}$, Liangyan Zheng ${ }^{2, *}$, Xin $\mathrm{Xu}^{1, *}$, Qiang Liu ${ }^{1}$, Jun $\mathrm{Ji}^{1, \bigotimes}$, \\ Lunguang $\mathrm{Yao}^{1}$, Yunchao Kan ${ }^{1}$, Yingzuo $\mathrm{Bi}^{3}$ \\ ${ }^{1}$ Henan Provincial Engineering Laboratory of Insects Bio-reactor, \\ Henan Provincial Engineering and Technology Center of Health Products for Livestock and Poultry, \\ Nanyang Normal University, Nanyang, 473061, PR China \\ ${ }^{2}$ Chengdu Agricultural College, Chengdu, 611130, PR China \\ ${ }^{3}$ College of Animal Science, South China Agricultural University, Guangzhou 510642, PR China \\ jijun020@126.com
}

Received: June 11, $2020 \quad$ Accepted: October 26, 2020

\begin{abstract}
Introduction: Canine parvovirus type-2 (CPV-2) causes acute infectious diseases in puppies, which show high morbidity and mortality. Better effect of vaccination against these diseases could be achieved with deeper knowledge of CPV-2 genotype dissemination and mutation history. This study investigated CPV-2-positive samples collected recently over a wide region of China. Material and Methods: A total of 118 faecal samples from dogs identified as CPV-positive were collected from veterinary clinics in central and eastern China. Overall, 16 strains collected from Anhui, 29 from Henan, and 16 from Zhejiang Province were sequenced to determine the genotypic composition of CPV-2 and mutational complexity of CPV-VP2. Results: The CPV-2a, CPV-2b, and CPV-2c genotypes were detected in Anhui and Henan Provinces, while CPV-2c alone was detected in Zhejiang Province. Sequence analysis of all strains showed $98.5 \%-99.8 \%, 98.3 \%-99.9 \%$, and $98.7 \%-99.8 \%$ identity among the 16 Anhui, 29 Henan, and 16 Zhejiang strains, respectively. Strains collected from Anhui and Henan Provinces showed lower identity (97.0\%), suggesting greater genetic divergence in central China. The mutation rates of Henan and Anhui strains were lower than that of Zhejiang strains. Major amino acid mutations occurred at sites 5, 370, 426, and 440. Epitope and entropy analyses implied these sites' likely conformance to the principles of mutation tendency, complexity, and diversity. Conclusion: The findings for the evolutionary structure of CPV-2 strains collected from three provinces in central and eastern China advance trend monitoring of the genetic variation in canine parvovirus and point to its implications in the development of novel vaccines.
\end{abstract}

Keywords: amino acid mutation site, canine parvovirus, phylogenetic tree, VP2 identity.

\section{Introduction}

Canine parvovirus (CPV) emerged in the late 1970s as a host-range variant of feline panleukopenia virus (FPV) (21). CPV belongs to the Parvoviridae family and is a nonenveloped virus with a single-stranded DNA genome (5,000 nt) comprising two open reading frames (ORFs). The first ORF encodes two nonstructural proteins, NS1 and NS2; the second ORF encodes two structural proteins, VP1 and VP2 (31). Since its discovery, this virus has been detected worldwide, primarily as $\mathrm{CPV}$ type-2 (CPV-2), and has become one of the most serious infectious pathogens among dogs. CPV-2 is among the most common aetiological agents of severe gastroenteritis, particularly in 6-20-week-old puppies, unvaccinated puppies, or puppies with poor maternal protection through passive immunity, and replicates mainly in intestinal crypts and the lymphoid organs but may reach any organ in susceptible animals. The most characteristic signs of this illness are diarrhoea, emesis, anorexia, depression, pyrexia, or hypothermia $(5,7,32)$.

Currently, only CPV-2 has been thoroughly investigated. The VP2 capsid protein, accounting for $90 \%$ of the CPV nucleocapsid and 426 amino acids in size, plays a crucial role in the structure of this virus and serves as the main protective antigen - two characteristics which are the classification criteria for 
CPV viral typing. Owing to variations in the antigenicity of VP2 across strains, multiple genotypes have emerged, including CPV-2a, CPV-2b, and CPV-2c. CPV-2a, CPV-2b, and CPV-2c differ at the $426^{\text {th }}$ amino acid (Asn in $2 \mathrm{a}$, Asp in 2b, and Glu in 2c) of the parvovirus VP2 protein $(11,17,18)$. In general, a novel CPV-2 variant replaces the old variants rapidly $(11,15)$. In recent decades, CPV-2c has become widespread in European countries $(6,11)$, the United States $(11,12)$, South America $(2,11,19,20)$, and Africa $(1,11,23)$. In Asia, CPV-2c was first reported from Vietnam in 2004 (11, 16); however, since then, this strain has not been prevalent in Asia $(4,11)$. Surprisingly, in the past few years, novel CPV-2c isolates from Asia have been identified in mainland China $(8,11,25,28)$, Taiwan (4, 11, 14), Laos $(11,24)$, and Thailand $(3,11)$. Therefore, in the present study, VP2 sequence analysis of strains collected from central and eastern China was performed to determine the mutation tendency of CPV-2 in infected dogs sampled from October 2018 to April 2019.

\section{Material and Methods}

Sample collection and DNA extraction. During the major CPV-2 infection seasons lasting from October 2018 to April 2019, 118 samples of canine faeces from pets confirmed to be CPV-positive using a colloidal gold strip test (Immunochromatographic Rapid Test Kit, BioNote, Hwaseong-si, Korea), were collected from pet hospitals in Henan, Anhui, and Zhejiang Provinces. Detailed clinical information was recorded for each sampled dog. Each faecal sample was suspended in phosphate-buffered saline, and virus isolation from feline kidney (F81) cells was performed as described previously (27). The virus and F81 cells were incubated until cytopathic effects were apparent. The buffer solution containing the virus was centrifuged, and then $500 \mu \mathrm{L}$ of the supernatant was inoculated in a cell culture flask of F81 and cultured in cell culture tank at $37^{\circ} \mathrm{C}$ for $2 \mathrm{~h}$. After that, all the liquid in the culture flask was poured out and replaced by $5 \mathrm{~mL}$ cell culture medium, and the culture was continued for 3 days to collect the virus until the $5^{\text {th }}$ passage, and the virus infection of F81 cells was detected. In the whole process of cell inoculation, on average 3 passages were completed. DNA was extracted from the infected faeces or F81 cells using the EasyPure Viral DNA/RNA Kit (TransGen Biotechnology, Inc., Beijing, China) according to the manufacturer's instructions. The extracted DNA samples were stored at $-80^{\circ} \mathrm{C}$.

Cloning of the VP2-coding sequence of CPV. The complete VP2 sequences of CPV-positive samples were amplified from the extracted viral DNA via PCR using a high-fidelity enzyme (TransGen Biotechnology, Inc.) and the primer pair of CPV-F (5'-AGAGACAAT CTTGCACCAAT-3') and CPV-R (5'-ATGTTAATA TAATTTTCTAGGTGCT-3') (from nucleotides 27614536 of the sequence deposited at GenBank under accession number MF805797) was designed using
Primer Premier 5.0 software (Premier Biosoft, San Francisco, CA, USA). Each amplicon was then analysed by electrophoresis, and the amplified VP2 sequences were cloned and sequenced. The vaccination status of each dog from which the samples was noted from records, as was the specific vaccine strain administered. That was the Vanguard Plus 5 classical CPV-2 commercial vaccine strain (Pfizer Inc., Lincoln, NE, USA).

Characterisation and genotyping of CPV-2. Sequences were assembled by overlapping and analysed using the SeqMan module of Lasergene 7 (DNASTAR, Inc., Madison, WI, USA). The NCBI nucleotide database was searched for typical VP2 gene sequences to identify mutations in CPV-VP2 sequences in various regions of China in recent years. The sequences obtained were compared using the NCBI nucleotide database to analyse the genotypes.

Mutation and phylogenetic analyses of CPV-2. The VP2 sequences of the investigated strains were compared with those of reference strains using Clustal X (22) to analyse identity and amino acid sequence variation. The CPV-VP2 sequences tested in this study and reference sequences in GenBank were aligned using MEGA 7.0 (13), and phylogenetic trees were constructed to analyse relationships between the strains tested in this study and reference strains. The details of the reference sequences incorporated in the phylogenetic tree are shown in Table 1. Differences in nucleotide and amino acid sequences between the collected and reference strains from China and overseas were analysed using MegAlign (DNASTAR, Inc.). EvolView 2 (10) was adopted for visualising and annotating phylogenetic trees with geographic location and phylogenetic clustering. Sequence identity between the collected and reference strains was determined using Lasergene 7 to examine genotypic variations across different regions of the study area.

Epitope and selection pressure analyses of CPV-2. Antigenic epitopes of the CPV-2c VP2 protein were predicted using Lasergene 7. Selection pressure on CPV based on entropy was analysed using VP2 sequences of the 61 collected strains using BioEdit (9).

\section{Results}

Virus identification and genotypes. All collected samples tested positive for CPV on the basis of virus isolation and PCR assays. The complete sequence (1755 nt) of the VP2 structural protein of 61 strains was obtained from 118 strains tested and deposited in GenBank (where strains had 100\% sequence identity and were from the same site, only one was retained for analysis). Accession numbers and clinical information for each strain are summarised in Table 2. Sequence analysis of strains collected from the three provinces showed that the infection rates of CPV-2a, CPV-2b, and CPV-2c were $3 / 29,4 / 29$ and $22 / 29$, respectively, in Henan Province and $2 / 16,3 / 16$, and $11 / 16$, respectively, in Anhui Province; all CPV genotypes collected from 
Zhejiang Province were CPV-2c. The vaccination rate was 50\% in Anhui Province, 58.62\% in Henan Province, and $87.5 \%$ in Zhejiang Province. From the above distribution data, CPV-2c is evidently the predominant CPV genotype in central and eastern China. Genotypes and geographical distribution of the 61 strains are shown in Fig. 1.

Nucleotide identity of the VP2 gene. Comparison of the nucleotide acid sequences of the VP2 gene between the 61 strains obtained in this study and reference strains in GenBank revealed 98.3\%-99.99\% identity. The nucleotide identity was $98.5 \%-99.8 \%$ for the 16 strains collected from Anhui Province, 98.3\%-99.8\% for the 29 strains from Henan Province, and $98.7 \%-99.8 \%$ for the 16 Zhejiang Province strains. Between provinces, the identity was $97.0 \%-99.9 \%$ comparing strains collected in Anhui and Henan Provinces, 97.3\%-99.8\% for strains collected in Henan and Zhejiang Provinces, and 97.6\%-99.8\% when Anhui and Zhejiang Province strains were examined.

Table 1. Reference strains used in the study

\begin{tabular}{|c|c|c|c|c|}
\hline Strain & Accession number & Genotype & Place of isolation & Year of isolation \\
\hline nn171025 & MK332005 & CPV-2a & Guangxi & 2017 \\
\hline nn17101 & MK332003 & $\mathrm{CPV}-2 \mathrm{a}$ & Guangxi & 2017 \\
\hline nn1693 & MK332002 & $\mathrm{CPV}-2 \mathrm{~b}$ & Guangxi & 2016 \\
\hline nn1681 & MK331996 & CPV-2b & Guangxi & 2016 \\
\hline nn171105 & MK332007 & CPV-2c & Guangxi & 2017 \\
\hline nn171024 & MK332004 & CPV-2c & Guangxi & 2017 \\
\hline CPV-411b.us.9 & EU659121 & CPV-2b & The USA & 1998 \\
\hline CPV-13.us.81 & EU659118 & CPV-2a & The USA & 1981 \\
\hline CPV-6.us. 80 & EU659117 & CPV-2 & The USA & 1980 \\
\hline Raccoon/ WI/ 37/ 10 & JN867618 & CPV-2a & The USA & 2010 \\
\hline $110 / 07-27$ & FJ005236 & $\mathrm{CPV}-2 \mathrm{c}$ & The USA & 2007 \\
\hline $08-\mathrm{B}$ & GU362934 & CPV-2a & Italy & 2008 \\
\hline $260-00$ & MF177231 & CPV-2a & Italy & 2000 \\
\hline $140 / 05$ & FJ005265 & $\mathrm{CPV}-2 \mathrm{~b}$ & Italy & 2005 \\
\hline CPV /IZSSI /25835/ 09 & KU508407 & CPV-2c & Italy & 2009 \\
\hline $56 / 00$ & FJ222821 & $\mathrm{CPV}-2 \mathrm{c}$ & Italy & 2000 \\
\hline CPV/dog/HCM/20/2013 & LC216910 & CPV-2c & Indonesia & 2013 \\
\hline Pome & EF599098 & CPV-2c(a) & South Korea & 2007 \\
\hline DH326 & EF599097 & CPV-2b & South Korea & 2007 \\
\hline DH426 & EF599096 & CPV-2a & South Korea & 2007 \\
\hline $16 \mathrm{M} 130$ & MH643886 & CPV-2 & South Korea & 2016 \\
\hline 2670/CPV-2c/2010/Ind & KX425920 & CPV-2c & India & 2010 \\
\hline CU267 & MH711901 & CPV-2c & Thailand & 2017 \\
\hline TH011401 & KT364589 & CPV-2c & Thailand & 2014 \\
\hline $\mathrm{T} 37$ & CPU72698 & CPV-2a & Taiwan & 1996 \\
\hline $\mathrm{T} 10$ & CPU72696 & $\mathrm{CPV}-2 \mathrm{~b}$ & Taiwan & 1996 \\
\hline 2017090801 & MH127909 & CPV-2c & Taiwan & 2017 \\
\hline Protein (VP2) & KU244254 & CPV-2c & Taiwan & 2015 \\
\hline $\mathrm{PV} / \mathrm{PL} / \mathrm{HeN} 02 / 08$ & EU441280 & CPV-2a & Henan & 2008 \\
\hline Henan 42 & KJ438805 & CPV-2a & Henan & 2013 \\
\hline CPV-HN1617 & MF467229 & $\mathrm{CPV}-2 \mathrm{c}$ & Henan & 2016 \\
\hline CPV-zj18 & KM386948 & CPV-2b & Zhejiang & 2014 \\
\hline CPV-zj7 & KM386937 & CPV-2a & Zhejiang & 2014 \\
\hline Beijing & HQ883267 & CPV-2a & Beijing & 2010 \\
\hline BJ-1 & MN101726 & CPV-2a & Beijing & 2018 \\
\hline 2011-BJ-B43 & KF803527 & CPV-2b & Beijing & 2011 \\
\hline 2011-BJ-B6 & KF803606 & CPV-2b & Beijing & 2011 \\
\hline CPV-SH1516 & MG013488 & CPV-2c & Shanghai & 2017 \\
\hline Shanghai/04g/2016 & KY937646 & CPV-2a & Shanghai & 2016 \\
\hline ShangHai/3-2/2016 & KY937640 & CPV-2a & Shanghai & 2016 \\
\hline Shanghai/03g/2016 & KY937637 & CPV-2c & Shanghai & 2016 \\
\hline CPVpf/2007(vaccine) & FJ197847 & CPV-2 & South Korea & 2007 \\
\hline 29/97(vaccine) & FJ222823 & CPV-2b & N.I. ${ }^{\mathrm{a}}$ & 2008 \\
\hline CPV-GX1581 & MF467242 & $\mathrm{CPV}-2 \mathrm{c}$ & Guangxi & 2015 \\
\hline
\end{tabular}

${ }^{a}$ no information 
Table 2. Clinical information on sources of Chinese canine parvovirus type 2 (CPV-2) obtained in this study

\begin{tabular}{|c|c|c|c|c|c|c|c|c|}
\hline Strain & Genotype & Site & $\begin{array}{l}\text { Date } \\
\text { of sampling }\end{array}$ & Age & Breed & Sex & $\begin{array}{l}\text { Vaccinated } \\
\text { status }\end{array}$ & Accession number \\
\hline CH-AH-D1 & CPV-2b & Hefei & Oct. 7, 2018 & 5 months & Poodle & Female & Unvaccinated & MN119560 \\
\hline CH-AH-D2 & CPV-2c & Anqing & Oct. 7, 2018 & 2 years & Poodle & Male & 1 dose & MN119561 \\
\hline CH-AH-D3 & CPV-2c & Suzhou & Oct. 8,2018 & 1 year & Mixed & Female & 2 doses & MN119562 \\
\hline CH-AH-D4 & CPV-2c & Suzhou & Oct. 11, 2018 & 3 months & Poodle & Male & Unvaccinated & MN119563 \\
\hline CH-AH-D5 & $\mathrm{CPV}-2 \mathrm{~b}$ & Wuhu & Nov. 9, 2018 & 1 year & Mixed & Female & 2 doses & MN119564 \\
\hline CH-AH-D6 & CPV-2c & Anqing & Nov. 11, 2018 & 4 months & Poodle & Female & 1 dose & MN119565 \\
\hline CH-AH-D7 & CPV-2c & Suzhou & Nov. 13, 2018 & 45 days & Schnauzer & Male & Unvaccinated & MN119566 \\
\hline CH-AH-D8 & CPV-2c & Hefei & Nov. 30, 2018 & 7 months & Mixed & Male & 1 dose & MN119567 \\
\hline CH-AH-D9 & CPV-2c & Wuhu & Dec. 1,2018 & 6 months & Retriever & Male & Unvaccinated & MN119568 \\
\hline CH-AH-D10 & CPV-2c & Suzhou & Dec. 3, 2018 & 3 months & Schnauzer & Female & Unvaccinated & MN119569 \\
\hline CH-AH-D11 & CPV-2c & Anqing & Jan. 7, 2019 & 8 months & Mixed & Male & 2 doses & MN119570 \\
\hline CH-AH-D12 & CPV-2c & Suzhou & Feb. 7, 2019 & 4 months & Mixed & Female & Unvaccinated & MN119571 \\
\hline CH-AH-D13 & CPV-2c & Wuhu & Mar. 11, 2019 & 2 months & Poodle & Female & 1 dose & MN119572 \\
\hline CH-AH-D14 & CPV-2a & Hefei & Mar. 16, 2019 & 52 days & Mixed & Female & Unvaccinated & MN119573 \\
\hline CH-AH-D15 & CPV-2a & Anqing & Apr. 2, 2019 & 4 months & Retriever & Male & 1 dose & MN119574 \\
\hline CH-AH-D16 & CPV-2b & Suzhou & Apr. 3, 2019 & 3 months & Poodle & Female & Unvaccinated & MN119575 \\
\hline CH-HN-D1 & CPV-2c & Zhengzhou & Oct. 3,2018 & 40 days & Mixed & Male & Unvaccinated & MN119576 \\
\hline CH-HN-D2 & CPV-2c & Hebi & Oct. 4, 2018 & 4 months & Mixed & Female & 1 dose & MN119577 \\
\hline CH-HN-D3 & CPV-2c & Nanyang & Oct. 8, 2018 & 10 months & Mixed & Male & 1 dose & MN119578 \\
\hline CH-HN-D4 & CPV-2c & Nanyang & Nov. 6, 2018 & 5 months & Retriever & Female & 1 dose & MN119579 \\
\hline CH-HN-D5 & CPV-2b & Luoyang & Nov. 7, 2018 & 4 months & Mixed & Male & Unvaccinated & MN119580 \\
\hline CH-HN-D6 & CPV-2c & Xinxiang & Nov. 12,2018 & 5 months & Schnauzer & Male & Unvaccinated & MN119581 \\
\hline CH-HN-D7 & CPV-2a & Xinyang & Nov. 14, 2018 & 4 months & Mixed & Female & 1 dose & MN119582 \\
\hline CH-HN-D8 & CPV-2c & Luoyang & Nov. 16, 2018 & 2 months & Mixed & Female & 1 dose & MN119583 \\
\hline CH-HN-D9 & CPV-2c & Zhengzhou & Nov. 28, 2018 & 2 months & Schnauzer & Male & Unvaccinated & MN119584 \\
\hline CH-HN-D10 & CPV-2c & Anyang & Nov. 29, 2018 & 4 months & Mixed & Female & 1 dose & MN119585 \\
\hline CH-HN-D11 & CPV-2c & Xinyang & Dec. 3,2018 & 50 days & Mixed & Female & 1 dose & MN119586 \\
\hline CH-HN-D12 & CPV-2c & Nanyang & Jan. 1, 2019 & 3 months & Retriever & Male & Unvaccinated & MN119587 \\
\hline CH-HN-D13 & CPV-2c & Shangqiu & Jan. 3, 2019 & 3 months & Mixed & Female & 1 dose & MN119588 \\
\hline CH-HN-D14 & CPV-2a & Anyang & Jan. 4, 2019 & 4 months & Mixed & Female & 1 dose & MN119589 \\
\hline CH-HN-D15 & CPV-2a & Zhengzhou & Jan. 5, 2019 & 1 month & Mixed & Male & Unvaccinated & MN119590 \\
\hline CH-HN-D16 & CPV-2b & Anyang & Jan. 5, 2019 & 2 months & Poodle & Male & Unvaccinated & MN119591 \\
\hline CH-HN-D17 & CPV-2c & Zhengzhou & Feb. 3, 2019 & 1 year & Mixed & Female & 2 doses & MN119592 \\
\hline CH-HN-D18 & CPV-2b & Nanyang & Feb. 4, 2019 & 4 months & Poodle & Male & Unvaccinated & MN119593 \\
\hline CH-HN-D19 & CPV-2c & Xinxiang & Feb. 5, 2019 & 5 months & Mixed & Female & 1 dose & MN119594 \\
\hline CH-HN-D20 & CPV-2b & Hebi & Mar. 8, 2019 & 2 months & Poodle & Female & Unvaccinated & MN119595 \\
\hline CH-HN-D21 & CPV-2c & Xinxiang & Mar. 9, 2019 & 5 months & Mixed & Male & 1 dose & MN119596 \\
\hline CH-HN-D22 & CPV-2c & Nanyang & Mar. 10, 2019 & 8 months & Retriever & Male & 2 doses & MN119597 \\
\hline CH-HN-D23 & CPV-2c & Hebi & Mar. 12, 2019 & 3 months & Mixed & Male & 1 dose & MN119598 \\
\hline CH-HN-D24 & CPV-2c & Luoyang & Mar. 15, 2019 & 2 months & Mixed & Female & 1 dose & MN119599 \\
\hline CH-HN-D25 & CPV-2c & Anyang & Mar. 29, 2019 & 2 months & Schnauzer & Female & Unvaccinated & MN119600 \\
\hline CH-HN-D26 & CPV-2c & Zhengzhou & Mar. 31, 2019 & 4 months & Mixed & Male & Unvaccinated & MN119601 \\
\hline CH-HN-D27 & CPV-2c & Luoyang & Apr. 1, 2019 & 1 year & Mixed & Female & 1 dose & MN119602 \\
\hline CH-HN-D28 & CPV-2c & Nanyang & Apr. 4, 2019 & 9 months & Mixed & Female & 1 dose & MN119603 \\
\hline CH-HN-D29 & CPV-2c & Zhengzhou & Apr. 5, 2019 & 4 months & Poodle & Male & Unvaccinated & MN119604 \\
\hline CH-ZJ-D1 & CPV-2c & Huzhou & Oct. 2, 2018 & 1 month & Mixed & Female & 1 dose & MN119605 \\
\hline CH-ZJ-D2 & CPV-2c & Hangzhou & Oct. 3,2018 & 3 months & Mixed & Male & Unvaccinated & MN119606 \\
\hline CH-ZJ-D3 & CPV-2c & Jinhua & Oct. 4, 2018 & 2 months & Mixed & Female & 1 dose & MN119607 \\
\hline CH-ZJ-D4 & CPV-2c & Ningbo & Oct. 8,2018 & 4 months & Retriever & Male & 1 dose & MN119608 \\
\hline CH-ZJ-D5 & CPV-2c & Hangzhou & Nov. 10, 2018 & 3 months & Mixed & Male & 1 dose & MN119609 \\
\hline CH-ZJ-D6 & CPV-2c & Ningbo & Nov. 11, 2018 & 6 months & Mixed & Female & 2 doses & MN119610 \\
\hline CH-ZJ-D7 & CPV-2c & Hangzhou & Nov. 17, 2018 & 2 months & Mixed & Female & Unvaccinated & MN119611 \\
\hline CH-ZJ-D8 & CPV-2c & Jinhua & Dec. 1,2018 & 5 months & Mixed & Male & 1 dose & MN119612 \\
\hline CH-ZJ-D9 & CPV-2c & Huzhou & Dec. 1,2018 & 3 months & Mixed & Male & 1 dose & MN119613 \\
\hline CH-ZJ-D10 & CPV-2c & Shaoxing & Dec. 3,2018 & 4 months & Retriever & Female & 1 dose & MN119614 \\
\hline CH-ZJ-D11 & CPV-2c & Jinhua & Jan. 4, 2019 & 7 months & Mixed & Male & 2 doses & MN119615 \\
\hline CH-ZJ-D12 & CPV-2c & Hangzhou & Feb. 6, 2019 & 2 months & Mixed & Male & 1 dose & MN119616 \\
\hline CH-ZJ-D13 & CPV-2c & Jinhua & Mar. 14, 2019 & 3 months & Mixed & Male & 1 dose & MN119617 \\
\hline CH-ZJ-D14 & CPV-2c & Shaoxing & Mar. 14, 2019 & 1 year & Mixed & Female & 1 dose & MN119618 \\
\hline CH-ZJ-D15 & CPV-2c & Hangzhou & Apr. 4, 2019 & 8 months & Mixed & Male & 2 doses & MN119619 \\
\hline CH-ZJ-D16 & CPV-2c & Jinhua & Apr. 4, 2019 & 9 months & Retriever & Female & 2 doses & MN119620 \\
\hline
\end{tabular}


Table 3. Statistics of the main amino acid mutation sites in the VP2 capsid protein of canine parvovirus type 2 in Chinese and reference strains

\begin{tabular}{|c|c|c|c|c|c|c|}
\hline \multirow{2}{*}{ Strains/GenBank Accession number } & \multicolumn{6}{|c|}{ Mutation sites: amino acid residue } \\
\hline & 5 & 30 & 130 & 370 & 426 & 440 \\
\hline EU659117-2/the USA/1980 & A & $\mathrm{G}$ & $\mathrm{V}$ & $\mathrm{Q}$ & $\mathrm{N}$ & $\mathrm{T}$ \\
\hline FJ197847-2/South Korea/2007/Vaccine & 1 & 1 & 1 & 1 & 1 & 1 \\
\hline MH643886-2/South Korea/2016 & 1 & 1 & 1 & 1 & 1 & 1 \\
\hline EU659118-2a/the USA/1981 & 1 & 1 & 1 & 1 & 1 & 1 \\
\hline MF177231-2a/Italy/2000 & 1 & 1 & 1 & 1 & 1 & 1 \\
\hline GU362934-2a/Italy/2008 & 1 & 1 & 1 & 1 & 1 & 1 \\
\hline EU441280-2a/Henan/2008 & 1 & 1 & 1 & 1 & 1 & 1 \\
\hline HQ883267-2a/Beijing/2010 & 1 & 1 & 1 & 1 & 1 & $\mathrm{~A}$ \\
\hline KJ438805-2a/Henan/2013 & 1 & 1 & 1 & 1 & 1 & A \\
\hline KY937646-2a/Shanghai/2016 & 1 & 1 & 1 & 1 & 1 & A \\
\hline MK332005-2a/Guangxi/2017 & 1 & 1 & 1 & 1 & 1 & $\mathrm{~A}$ \\
\hline MN101726-2a/Beijing/2018 & 1 & 1 & 1 & 1 & 1 & $\mathrm{~A}$ \\
\hline CPU72696-2b/Taiwan/1996 & 1 & 1 & 1 & 1 & $\mathrm{D}$ & 1 \\
\hline EU659121-2b/the USA/1998 & 1 & 1 & 1 & 1 & $\mathrm{D}$ & 1 \\
\hline FJ005265-2b/Italy/2005 & 1 & 1 & 1 & 1 & $\mathrm{D}$ & 1 \\
\hline KF803606-2b/Beijing/2011 & 1 & 1 & 1 & 1 & $\mathrm{D}$ & 1 \\
\hline MK332002-2b/Guangxi/2016 & 1 & 1 & 1 & 1 & $\mathrm{D}$ & A \\
\hline MK331996-2b/Guangxi/2016 & 1 & 1 & 1 & 1 & $\mathrm{D}$ & $\mathrm{A}$ \\
\hline KU508407-2c/Italy/2009 & 1 & 1 & 1 & 1 & $\mathrm{E}$ & $\backslash$ \\
\hline KX425920-2c/India/2010 & 1 & 1 & 1 & 1 & $\mathrm{E}$ & 1 \\
\hline KU244254-2c/Taiwan/2015 & 1 & 1 & 1 & 1 & $\mathrm{E}$ & 1 \\
\hline LC216910-2c/Indonesia/2013 & $\mathrm{G}$ & 1 & 1 & $\mathrm{R}$ & $\mathrm{E}$ & 1 \\
\hline MF467242-2c/Vaccine/2015 & $\mathrm{G}$ & 1 & 1 & $\mathrm{R}$ & $\mathrm{E}$ & 1 \\
\hline MF467229-2c/Henan/2016 & $\mathrm{G}$ & 1 & 1 & $\mathrm{R}$ & $\mathrm{E}$ & 1 \\
\hline MGo13488-2c/Shanghai/2017 & $\mathrm{G}$ & 1 & 1 & $\mathrm{R}$ & $\mathrm{E}$ & 1 \\
\hline CH-AH-D1 & $\mathrm{G}$ & 1 & 1 & 1 & $\mathrm{D}$ & $\mathrm{A}$ \\
\hline CH-AH-D2 & 1 & 1 & 1 & $\mathrm{R}$ & $\mathrm{E}$ & 1 \\
\hline CH-AH-D3 & 1 & W & 1 & $\mathrm{R}$ & $\mathrm{E}$ & 1 \\
\hline CH-AH-D4 & $\mathrm{G}$ & 1 & 1 & $\mathrm{R}$ & $\mathrm{E}$ & 1 \\
\hline CH-AH-D5 & 1 & 1 & 1 & 1 & $\mathrm{D}$ & $\mathrm{A}$ \\
\hline CH-AH-D6 & G & 1 & 1 & $\mathrm{R}$ & $\mathrm{E}$ & 1 \\
\hline CH-AH-D7 & 1 & 1 & 1 & $\mathrm{R}$ & $\mathrm{E}$ & 1 \\
\hline CH-AH-D8 & 1 & 1 & 1 & $\mathrm{R}$ & $\mathrm{E}$ & 1 \\
\hline CH-AH-D9 & $\mathrm{G}$ & 1 & 1 & $\mathrm{R}$ & $\mathrm{E}$ & 1 \\
\hline CH-AH-D10 & G & 1 & 1 & $\mathrm{R}$ & $\mathrm{E}$ & 1 \\
\hline CH-AH-D11 & G & 1 & 1 & $\mathrm{R}$ & $\mathrm{E}$ & 1 \\
\hline CH-AH-D12 & G & 1 & 1 & $\mathrm{R}$ & $\mathrm{E}$ & 1 \\
\hline CH-AH-D13 & $\mathrm{G}$ & 1 & 1 & $\mathrm{R}$ & E & 1 \\
\hline CH-AH-D14 & 1 & 1 & 1 & 1 & 1 & $\mathrm{~A}$ \\
\hline CH-AH-D15 & 1 & 1 & 1 & 1 & 1 & $\mathrm{~A}$ \\
\hline CH-AH-D16 & 1 & 1 & 1 & 1 & $\mathrm{D}$ & $\mathrm{A}$ \\
\hline CH-HN-D1 & $\mathrm{G}$ & 1 & 1 & $\mathrm{R}$ & $\mathrm{E}$ & 1 \\
\hline CH-HN-D2 & G & 1 & 1 & $\mathrm{R}$ & $\mathrm{E}$ & 1 \\
\hline CH-HN-D3 & G & 1 & 1 & $\mathrm{R}$ & $\mathrm{E}$ & 1 \\
\hline CH-HN-D4 & $\mathrm{G}$ & 1 & 1 & $\mathrm{R}$ & $\mathrm{E}$ & 1 \\
\hline CH-HN-D5 & 1 & 1 & 1 & 1 & $\mathrm{D}$ & $\mathrm{A}$ \\
\hline CH-HN-D6 & $\mathrm{G}$ & 1 & 1 & $\mathrm{R}$ & $\mathrm{E}$ & 1 \\
\hline CH-HN-D7 & 1 & 1 & A & 1 & 1 & $\mathrm{~A}$ \\
\hline CH-HN-D8 & 1 & 1 & 1 & $\mathrm{R}$ & $\mathrm{E}$ & 1 \\
\hline CH-HN-D9 & G & 1 & 1 & $\mathrm{R}$ & $\mathrm{E}$ & 1 \\
\hline CH-HN-D10 & $\mathrm{G}$ & 1 & 1 & $\mathrm{R}$ & $\mathrm{E}$ & 1 \\
\hline CH-HN-D11 & $\mathrm{G}$ & 1 & 1 & $\mathrm{R}$ & $\mathrm{E}$ & 1 \\
\hline CH-HN-D12 & $\mathrm{G}$ & 1 & 1 & $\mathrm{R}$ & E & 1 \\
\hline CH-HN-D13 & G & 1 & 1 & $\mathrm{R}$ & $\mathrm{E}$ & 1 \\
\hline CH-HN-D14 & 1 & 1 & 1 & 1 & 1 & $\mathrm{~A}$ \\
\hline CH-HN-D15 & 1 & 1 & 1 & 1 & 1 & $\mathrm{~A}$ \\
\hline
\end{tabular}


Table 3 (continued)

\begin{tabular}{|c|c|c|c|c|c|c|}
\hline CH-HN-D16 & 1 & 1 & 1 & 1 & $\mathrm{D}$ & $\mathrm{A}$ \\
\hline CH-HN-D17 & G & 1 & 1 & $\mathrm{R}$ & $\mathrm{E}$ & 1 \\
\hline CH-HN-D18 & 1 & 1 & 1 & 1 & $\mathrm{D}$ & A \\
\hline CH-HN-D19 & G & 1 & 1 & $\mathrm{R}$ & $\mathrm{E}$ & 1 \\
\hline CH-HN-D20 & 1 & 1 & 1 & 1 & $\mathrm{D}$ & A \\
\hline CH-HN-D21 & G & 1 & 1 & $\mathrm{R}$ & $\mathrm{E}$ & 1 \\
\hline CH-HN-D22 & G & 1 & 1 & $\mathrm{R}$ & $\mathrm{E}$ & 1 \\
\hline CH-HN-D23 & G & 1 & 1 & $\mathrm{R}$ & $\mathrm{E}$ & 1 \\
\hline CH-HN-D24 & G & 1 & 1 & $\mathrm{R}$ & $\mathrm{E}$ & 1 \\
\hline CH-HN-D25 & G & 1 & 1 & $\mathrm{R}$ & $\mathrm{E}$ & 1 \\
\hline CH-HN-D26 & G & 1 & 1 & $\mathrm{R}$ & $\mathrm{E}$ & 1 \\
\hline CH-HN-D27 & G & 1 & 1 & $\mathrm{R}$ & $\mathrm{E}$ & 1 \\
\hline CH-HN-D28 & G & 1 & 1 & $\mathrm{R}$ & $\mathrm{E}$ & 1 \\
\hline CH-HN-D29 & $\mathrm{G}$ & 1 & 1 & $\mathrm{R}$ & $\mathrm{E}$ & 1 \\
\hline CH-ZJ-D1 & G & 1 & 1 & $\mathrm{R}$ & $\mathrm{E}$ & 1 \\
\hline CH-ZJ-D2 & G & 1 & 1 & $\mathrm{R}$ & $\mathrm{E}$ & 1 \\
\hline CH-ZJ-D3 & G & 1 & 1 & $\mathrm{R}$ & $\mathrm{E}$ & 1 \\
\hline CH-ZJ-D4 & G & 1 & 1 & $\mathrm{R}$ & $\mathrm{E}$ & 1 \\
\hline CH-ZJ-D5 & G & 1 & 1 & $\mathrm{R}$ & $\mathrm{E}$ & 1 \\
\hline CH-ZJ-D6 & G & 1 & 1 & $\mathrm{R}$ & $\mathrm{E}$ & 1 \\
\hline CH-ZJ-D7 & G & 1 & 1 & $\mathrm{R}$ & $\mathrm{E}$ & 1 \\
\hline CH-ZJ-D8 & G & 1 & 1 & $\mathrm{R}$ & $\mathrm{E}$ & 1 \\
\hline CH-ZJ-D9 & G & 1 & 1 & $\mathrm{R}$ & $\mathrm{E}$ & 1 \\
\hline CH-ZJ-D10 & G & 1 & 1 & $\mathrm{R}$ & $\mathrm{E}$ & 1 \\
\hline CH-ZJ-D11 & $\mathrm{G}$ & 1 & 1 & $\mathrm{R}$ & $\mathrm{E}$ & 1 \\
\hline CH-ZJ-D12 & G & 1 & 1 & $\mathrm{R}$ & $\mathrm{E}$ & 1 \\
\hline CH-ZJ-D13 & G & 1 & 1 & $\mathrm{R}$ & $\mathrm{E}$ & 1 \\
\hline CH-ZJ-D14 & G & 1 & 1 & $\mathrm{R}$ & $\mathrm{E}$ & 1 \\
\hline CH-ZJ-D15 & G & 1 & 1 & $\mathrm{R}$ & $\mathrm{E}$ & 1 \\
\hline CH-ZJ-D16 & $\mathrm{G}$ & 1 & 1 & $\mathrm{R}$ & $\mathrm{E}$ & 1 \\
\hline
\end{tabular}

Strains with designations starting with "CH" are Chinese strains obtained in this study. (A:Ala, D:Asp, E: Glu, G: Gly, N:Asn, Q:Gln, R:Arg, T:Thr, V: Val, W:Try)

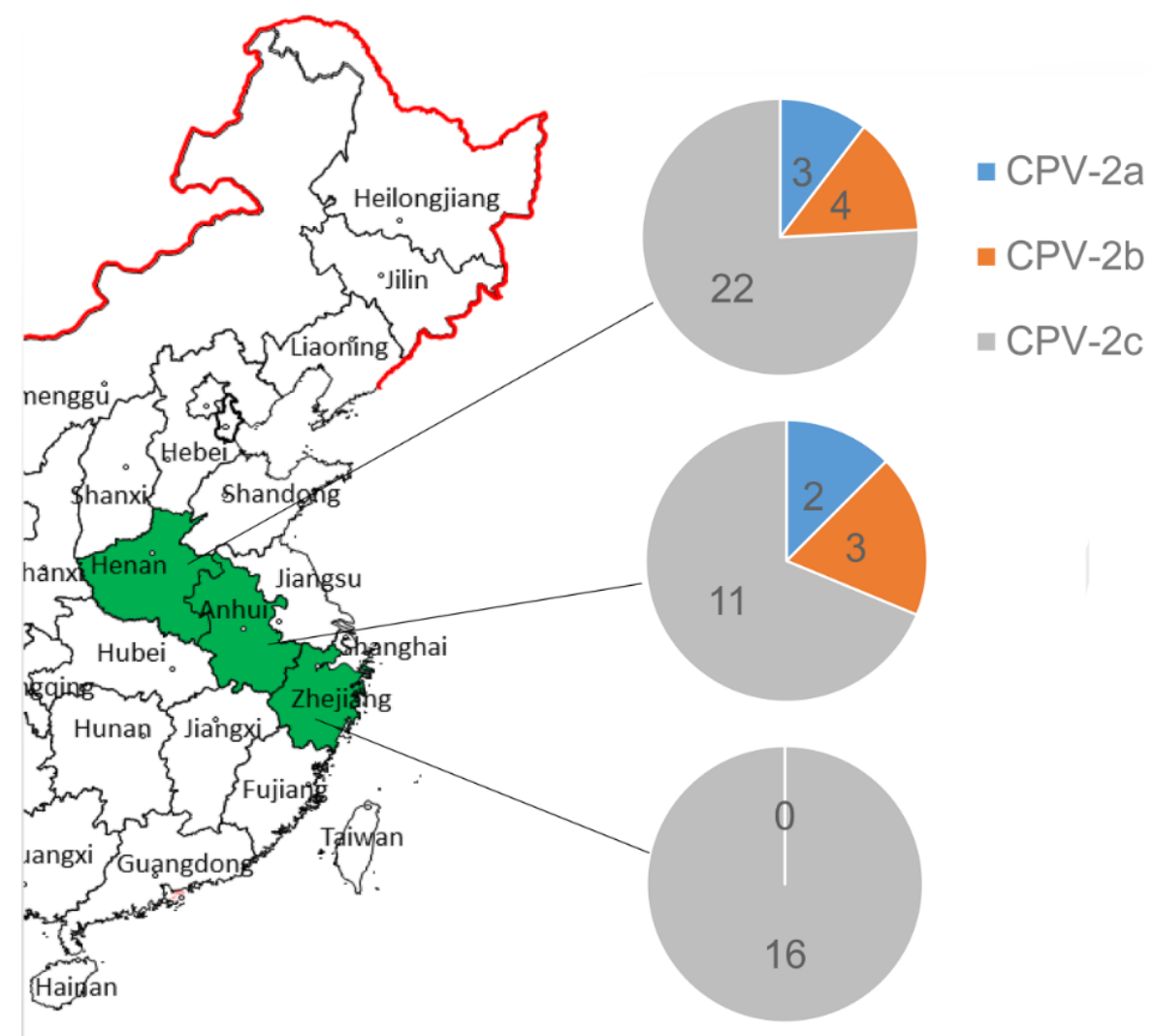

Fig. 1. Genotypes and geographical distribution of the canine parvovirus (CPV) strains tested in this study 


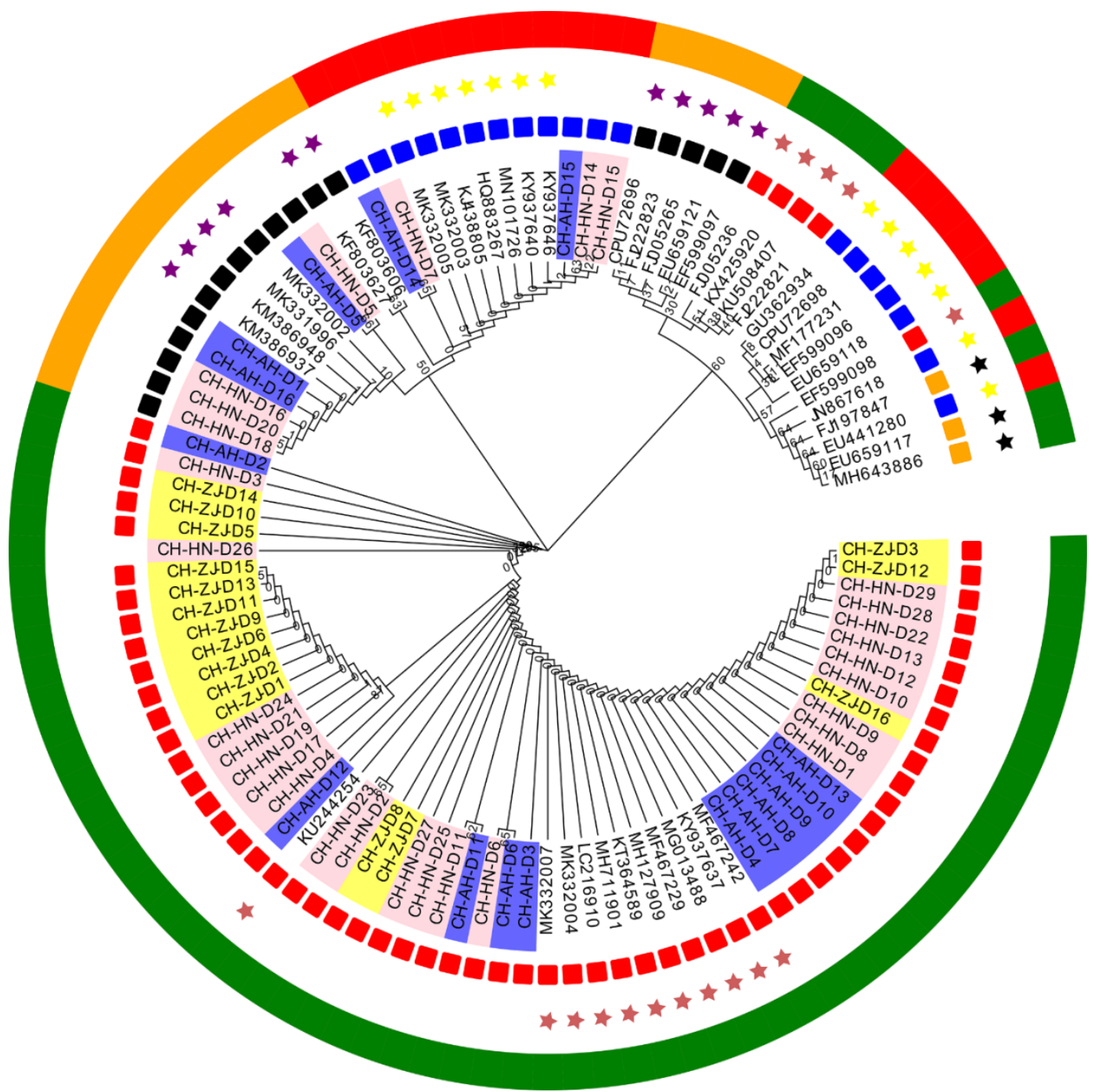

Fig. 2. Evolutionary tree of canine parvovirus type-2 (CPV-2). Blue areas represent the 16 strains collected from Anhui Province, pink areas represent the 29 strains collected from Henan Province, and yellow areas represent the 16 strains collected from Zhejiang Province. Yellow stars denote the CPV-2a reference strain; purple stars denote the CPV-2b reference strain; and red stars denote the $\mathrm{CPV}-2 \mathrm{c}$ reference strain. Red strips indicate the CPV-2a strain; orange strips indicate the CPV-2b strain; and green strips indicate the CPV-2c strain

A

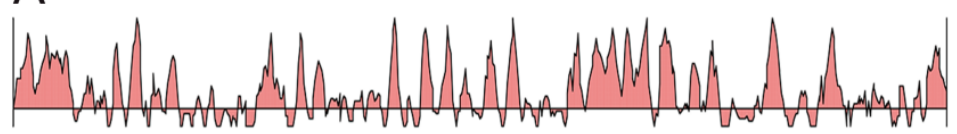

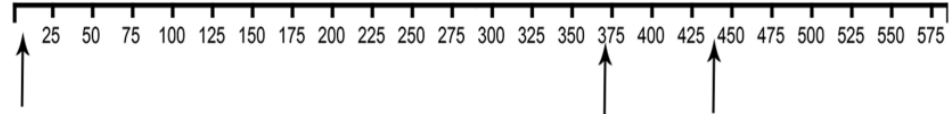

B

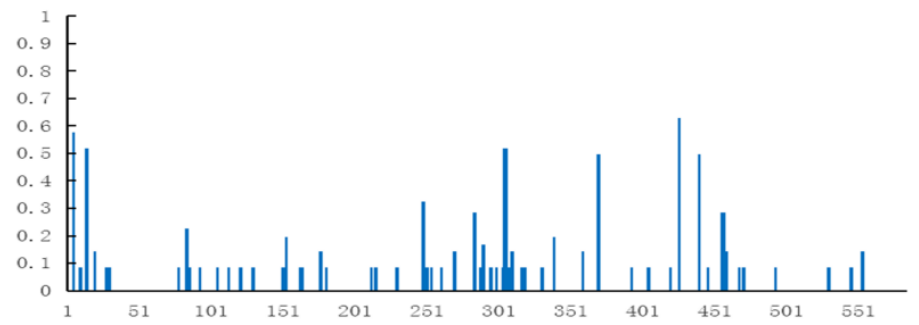

Fig. 3. Antigenic epitope prediction and amino acid entropy rates of the VP2 capsid protein of canine parvovirus type 2. A: Antigen epitope prediction for CPV-VP2; B: Amino acid entropy rates for CPV-VP2 
Main amino acid mutation sites of the VP2 protein. The main amino acid mutation sites of the VP2 protein of the 61 strains were 5, 30, 130, 370, 426, and 440. In CPV-2, Ala5Gly mutation was evident at a rate of $73.77 \%$ ( 8 strains in Anhui Province, 21 in Henan Province, and 16 in Zhejiang Province). Gly30Trp mutation occurred only in one strain (CH-AH-D3), and Val130Al mutation likewise (CH-HN-D7). Gln370Arg mutation was noted in $80.33 \%$ of isolates (11 strains in Anhui Province, 22 in Henan Province, and 16 in Zhejiang Province). Two mutations occurred at site 426, with a rate of $11.48 \%$ for Asn426Asp (three strains in Anhui and four in Henan Province) and $80.33 \%$ for Asn426Glu (11 strains in Anhui Province, 22 in Henan Province, and 16 in Zhejiang Province). Thr440Ala had mutated $80.33 \%$ of strains (11 in Anhui Province, 22 in Henan Province, and 16 in Zhejiang Province). The specific mutations by strain are presented in Table 3.

Phylogenetic and evolutionary relationships. A phylogenetic tree was constructed based on VP2 sequences of the 61 strains tested in this study and 45 representative reference strains. Compared with the strains collected from countries such as the United States, Italy, Japan, South Korea, and India, the reference strains collected from China were distantly related. Overall, $49 \mathrm{CPV}-2 \mathrm{c}$ strains $(80.33 \%)$ in this study were closely related to CPV-2c reference strains collected from Thailand, Indonesia, Taiwan, Shanghai, Guangxi, and Henan Provinces. Among these 49 strains, 11 were from Anhui Province, 22 from Henan Province, and 16 from Zhejiang Province. Seven strains collected from Anhui $(\mathrm{n}=3)$ and Henan $(\mathrm{n}=4)$ Provinces were similar to CPV-2b. Five strains were closely related to CPV-2a, two of which were from Anhui Province and three from Henan Province. The specific interrelationships between the collected and reference strains are depicted in Fig. 2.

Epitope and selection pressure analyses of CPV-VP2. Antigenic epitope analysis of the VP2 protein of CPV-2c predicted several epitopes mainly located at amino acid sites $0-25$ and 350-450 (Fig. 3A). In selection pressure analysis, the entropy was $>0.5$ mainly in two regions (0-51 and 251-451) (Fig. 3B). The results of epitope prediction and selection pressure analyses were consistent with one another.

\section{Discussion}

A CPV is widely distributed in various regions of China, and the virus undergoes rapid mutations. Since its identification, three important variations in the CPV genotype have occurred, resulting in the emergence of subtypes CPV-2a, CPV-2b, and CPV-2c. During 20092012, CPV-2a was the dominant genotype in the southern region of Nanjing (29). In this period and continuing until 2014, CPV-2c was not widespread in Henan Province (30). By 2015-2016, the novel CPV-2a strain had become the prevalent subtype in Henan,
Guangxi, and Jiangsu Provinces and in these years Aln5Gly and Gln370Arg mutations in genotype 2c were reported for the first time (26). In this study, the epidemiological trend of CPV-2 was observed from 2018 to 2019 through sequencing the CPV-2 strains collected from three provinces (Henan, Anhui and Zhejiang). Variations in the CPV-2 genotype were strongly affected by vaccination and tended to be due to 2c mutations. In the present study, trends of CPV infections in Anhui, Henan, and Zhejiang Provinces revealed $\mathrm{CPV}-2 \mathrm{c}$ as the most widespread genotype. Three genotypes coexisted in Anhui and Henan Provinces, which are not perfectly covered by the vaccination program. In Zhejiang Province, in contrast, the vaccination rate was high and $\mathrm{CPV}-2 \mathrm{c}$ was the only strain detected. Based on genotypic and immunisation history, CPV-2c shows high transmissibility and strong adaptability. It is therefore the genotype most likely to cause vaccination failure when the program uses classical CPV-2, and this genotype's dominance may be explained by its having evolved under pressure from immunisation.

The main amino acid mutations in the VP2 protein of the 61 strains occurred at sites 5, 370, 426, and 440 . In Anhui, Henan and Zhejiang Provinces, 45 (73.77\%) strains harboured a similar Ala5Gly mutation to the reference strain CPV-2c. This finding indicates that the amino acid mutation at site 5 may determine the CPV subtype (CPV-2c), with such mutations gradually becoming increasingly common in China and overseas. The nearly three quarters proportion of strains collected from Anhui, Henan and Zhejiang Provinces with the Ala5Gly mutation in the CPV-VP2 protein suggests that it has become prevalent. A total of $49(80.33 \%)$ strains in the three provinces displayed a similar Gln370Arg mutation to the reference strain CPV-2c. This site may alter the spatial structure of the VP2 protein to some extent, thereby affecting the pathogenicity of the virus (8). Mutations at the amino acid site 426 markedly affected the classification of CPV-2a, CPV-2b, and CPV-2c (30). The reference strain CPV-2a showed 426Asn, and mutation at this site occurred in 5 of the 61 strains $(8.20 \%)$ tested in this study. Seven $(11.48 \%)$ strains collected from Anhui and Henan Provinces were Asn426Asp-mutated in a similar way to the reference strain $\mathrm{CPV}-2 \mathrm{~b}$, although no mutation was detected in the strains collected from Zhejiang Province. A total of 49 $(80.33 \%)$ strains revealed a similar Asn426Gln mutation to the reference strain CPV-2c. These findings indicate that the Asn426Gln mutation was highly prevalent in the three provinces studied. Furthermore, the strains collected from Henan and Anhui Provinces (19.67\%) were like the reference strains CPV-2a and CPV-2b in having the Thr440Ala mutation. This result demonstrates that CPV strains carrying the Thr440Ala mutation are prevalent in Anhui and Henan Provinces. In terms of mutation rates, $\mathrm{CPV}-2 \mathrm{c}$ was the dominant mutant genotype in Anhui, Henan, and Zhejiang Provinces from October 2018 to April 2019. 
Sequence identities and phylogenetic relationships of the 61 strains collected from the three provinces revealed in this study will contribute to a better understanding of the frequency and mutation tendencies of CPV strains in these provinces. The VP2 sequences of the CPV strains collected from Anhui, Henan, and Zhejiang Provinces shared high identity without any outstanding variations; however, these strains showed relatively distant relationships with the classical vaccine strains. Moreover, there were no obvious regional differences in CPV genotype distribution according to the evolutionary tree. This result indicates that CPV genotypes are characterised by mutations at individual amino acid sites which do not affect the overall evolutionary characteristics of CPV.

In this study, mutations at four major amino acid sites led to the emergence of different genotypes, which further evolved into the highly immune-resistant subtype CPV-2c under pressure from immunisation as previously described $(4,8)$. The epitope is the antigenic sequence that stimulates B cells to produce antibodies, leading to antigen-antibody binding. The VP2 protein of CPV-2c presents multiple epitopes; therefore, multiple amino acid mutations might be triggered via adaptive selection for survival under pressure from immunisation. The present study provides a theoretical and technical basis for evolutionary analysis and vaccine strain selection against CPV-2c. Selection pressure analysis revealed that the genomic regions carrying mutations were complex, diverse, and highly prone to mutations, which further explains the differentiation of CPV-2 into additional genotypes in recent years (4). Two regions $(0-25$ and 350-450) in epitope prediction (Fig. 3A) and two regions (0-51 and 251-451) in selection pressure analysis (Fig. 3B) showed a high degree of coincidence in their entropy values. Moreover, the mutations at the sites $5,370,426$, and 440 in this study conform to the epidemic trends of the virus, further confirming the authenticity of the mutated sites in this study. Unfortunately, owing to the unavailability of specific sera for genotypic variants in our laboratory, we were unable to provide data for serological analysis in this study. Nonetheless, based on the results of epitope prediction and selection pressure analyses combined with vaccination history, the genotypic variations in strains collected from Anhui, Henan and Zhejiang Provinces in China may be attributed to pressure from immunisation. Animal inoculation experiments are needed to determine whether immunisation failure is a possible consequence of antibody pressure on evolution of CPV-2a, CPV-2b, and CPV-2c.

In conclusion, amino acid sequence analysis of the VP2 protein of $61 \mathrm{CPV}$ strains collected from central and eastern China indicated that $\mathrm{CPV}-2 \mathrm{c}$ is the predominant genotype of CPV in the study regions, particularly in Zhejiang Province. In addition, strains with CPV-2a and CPV-2b genotypes harbouring novel mutations were also detected. These results highlight the need for further research targeting different CPV genotypes to develop vaccines and establish more effective vaccination programs that increase the scope of immunisation.

Conflict of Interests Statement: The authors declare that there is no conflict of interests regarding the publication of this article.

Financial Disclosure Statement: This study was supported by the National Natural Science Foundation of China (Grant nos. 31802185 and 31870917), the Scientific and Technological Project of Henan Province (Grant no. 182107000040), the program for Innovative Research Teams of Science and Technology in the University of Henan Province (Grant no. 20IRTSTHN024), the Key Scientific and Technological Project of the Education Department of Henan province (Grant no. 18A230012), and Technological Project of Nanyang Normal University (Grant nos. 18046 and 2019QN009).

Animal Rights Statement: None required.

* These authors contributed equally to the article and should be considered co-first authors.

\section{References}

1. Amrani N., Desario C., Kadiri A., Cavalli A., Berrada J., Zro K., Sebbar G., Colaianni M.L., Parisi A., Elia G., Buonavoglia C., Malik J., Decaro N.: Molecular epidemiology of canine parvovirus in Morocco. Infect Genet Evol 2016, 41, 201-206, doi: 10.1016/j.meegid.2016.04.005.

2. Calderon M.G., Romanutti C., D'Antuono A., Keller L., Mattion N., La Torre J.: Evolution of canine parvovirus in Argentina between years 2003 and 2010: CPV2c has become the predominant variant affecting the domestic dog population. Virus Res 2011, 157, 106-110, doi: 10.1016/j.virusres.2011.02.015.

3. Charoenkul K., Tangwangvivat R., Janetanakit T., Boonyapisitsopa S., Bunpapong N., Chaiyawong S., Amonsin A.: Emergence of canine parvovirus type $2 \mathrm{c}$ in domestic dogs and cats from Thailand. Transbound Emerg Dis 2019, 66, 1518-1528, doi: 10.1111/tbed.13177.

4. Chiang S.Y., Wu H.Y., Chiou M.T., Chang M.C., Lin C.N.: Identification of a novel canine parvovirus type 2c in Taiwan. Virol J 2016, 13, 160, doi: 10.1186/s12985-016-0620-5.

5. De la Torre D., Mafla E., Puga B., Erazo L., Astolfi-Ferreira C., Ferreira A.P.: Molecular characterization of canine parvovirus variants (CPV-2a, CPV-2b, and CPV-2c) based on the VP2 gene in affected domestic dogs in Ecuador. Vet World 2018, 11, 480-487, doi: 10.14202/vetworld.2018.480-487.

6. Decaro N., Elia G., Martella V., Campolo M., Desario C., Camero M., Cirone F., Lorusso E., Lucente M.S., Narcisi D., Scalia P., Buonavoglia C.: Characterisation of the canine parvovirus type 2 variants using minor groove binder probe technology. J Virol Methods 2006, 133, 92-99, doi: 10.1016/j.jviromet.2005.10.026.

7. Figueiredo J., Miranda C., Souto R., Silva E., Fafetine J., Thompson G.: Genetic characterization of canine parvovirus type 2 subtypes in Maputo, Mozambique. Arch Microbiol 2017, 199, 543-549, doi: 10.1007/s00203-016-1320-7.

8. Geng Y., Guo D., Li C., Wang E., Wei S., Wang Z., Yao S., Zhao X., Su M., Wang X., Wang J., Wu R., Feng L., Sun D.: Co-Circulation of the Rare CPV-2c with Unique Gln370Arg Substitution, New CPV-2b with Unique Thr440Ala Substitution, 
and New CPV-2a with High Prevalence and Variation in Heilongjiang Province, Northeast China. PLoS One 2015, 10, e0137288, doi: 10.1371/journal.pone.0137288.

9. Hall T.A.: BioEdit: a user-friendly biological sequence alignemnt editor and analysis program for Windows 95/98/NT. Nucl Acids Symp Ser 1999, 41, 95-98.

10. He Z., Zhang H., Gao S., Lercher M.J., Chen W.-H., Hu S.: Evolview v2: an onlline visualization and management tool for customised and annotated phylogenetic trees. Nucleic Acids Res 2016, 44, W236-W241, doi: 10.1093/nar/gkw370.

11. Hoang M., Lin W.H., Le V.P., Nga B., Chiou M.T., Lin C.N.: Molecular epidemiology of canine parvovirus type 2 in Vietnam from November 2016 to February 2018. Virol J 2019, 16, 52, doi: 10.1186/s12985-019-1159-z.

12. Hong C., Decaro N., Desario C., Tanner P., Pardo M.C., Sanchez S., Buonavoglia C., Saliki J.T.: Occurrence of canine parvovirus type $2 \mathrm{c}$ in the United States. J Vet Diagn Invest 2007, 19, 535-539, doi: 10.1177/104063870701900512.

13. Kumar S., Stecher G., Tamura K.: MEGA7: Molecular Evolutionary Genetics Analysis version 7.0 for bigger datasets. Molecular Biology and Evolution 2016, 33, 1870-1874, doi: 10.1093/molbev/msw054.

14. Lin Y.C., Chiang S.Y., Wu H.Y., Lin J.H., Chiou M.T., Liu H.F., Lin C.N.: Phylodynamic and Genetic Diversity of Canine Parvovirus Type 2c in Taiwan. Int J Mol Sci 2017, 18, 1-11, doi: 10.3390/ijms 18122703.

15. Martella V., Cavalli A., Pratelli A., Bozzo G., Camero M., Buonavoglia D., Narcisi D., Tempesta M., Buonavoglia C.: A canine parvovirus mutant is spreading in Italy. $\mathrm{J}$ Clin Microbiol 2004, 42, 1333-1336, doi: 10.1128/jcm.42.3.1333-1336.2004.

16. Nakamura M., Tohya Y., Miyazawa T., Mochizuki M., Phung H.T., Nguyen N.H., Huynh L.M., Nguyen L.T., Nguyen P.N., Nguyen P.V., Nguyen N.P., Akashi H.: A novel antigenic variant of Canine parvovirus from a Vietnamese dog. Arch Virol 2004, 149, 2261-2269, doi: 10.1007/s00705-004-0367-y.

17. Parrish C.R., Aquadro C.F., Strassheim M.L., Evermann J.F., Sgro J.Y., Mohammed H.O.: Rapid antigenic-type replacement and DNA sequence evolution of canine parvovirus. J Virol 1991, 65, 6544-6552, doi: 10.1128/JVI.65.12.6544-6552.1991.

18. Parrish C.R., O'Connell P.H., Evermann J.F., Carmichael L.E.: Natural variation of canine parvovirus. Science 1985, 230, 1046-1048, doi: 10.1126/science.4059921.

19. Pedroza-Roldán C., Páez-Magallan V., Charles-Niño C., ElizondoQuiroga D., De Cervantes-Mireles R.L., López-Amezcua M.A.: Genotyping of Canine parvovirus in western Mexico. J Vet Diagn Invest 2015, 27, 107-111, doi: 10.1177/1040638714559969.

20. Pérez R., Francia L., Romero V., Maya L., López I., Hernández M.: First detection of canine parvovirus type $2 \mathrm{c}$ in South America. Vet Microbiol 2007, 124, 147-152, doi: 10.1016/j.vetmic. 2007.04.028.

21. Stucker K.M., Pagan I., Cifuente J.O., Kaelber J.T., Lillie T.D., Hafenstein S., Holmes E.C., Parrish C.R.: The role of evolutionary intermediates in the host adaptation of canine parvovirus. J Virol 2012, 86, 1514-1521, doi: 10.1128/JVI.06222-11.

22. Thompson J.D., Gibson T.J., Plewniak F., Jeanmougin F., Higgins D.G.: The Clustal_X Windows Interface: Flexible Strategies for Multiple Sequence Alignment Aided by Quality Analysis Tools. Nucleic Acids Res 1997, 25, 4876-4882, doi: 10.1093/nar/25.24.4876

23. Touihri L., Bouzid I., Daoud R., Desario C., El Goulli A.F., Decaro N., Ghorbel A., Buonavoglia C., Bahloul C.: Molecular characterization of canine parvovirus- 2 variants circulating in Tunisia. Virus Genes 2009, 38, 249-258, doi: 10.1007/s11262008-0314-1.

24. Vannamahaxay S., Vongkhamchanh S., Intanon M., Tangtrongsup S., Tiwananthagorn S., Pringproa K., Chuammitri P.: Molecular characterization of canine parvovirus in Vientiane, Laos. Arch Virol 2017, 162, 1355-1361, doi: 10.1007/s00705-016-3212-1.

25. Wang J., Lin P., Zhao H., Cheng Y., Jiang Z., Zhu H., Wu H., Cheng S.: Continuing evolution of canine parvovirus in China: Isolation of novel variants with an Ala5Gly mutation in the VP2 protein. Infect Genet Evol 2016, 38, 73-78, doi: 10.1016/j.meegid.2015.12.009.

26. Wu H., Li X., Wang L., Liu Y., Tian K..: Molecular epidemiological survey of canine parvovirus in domestic dogs in four provinces, China. Virusdisease 2018, 29, 113-117, doi: 10.1007/s13337-018-0427-7.

27. Yu Y., Su J., Wang J., Xi J., Mao Y., Hou Q., Zhang X., Liu W.: A rapid method for establishment of a reverse genetics system for canine parvovirus. Virus Genes 2017, 53, 876-882, doi:10.1007/s11262-017-1497-0.

28. Zhao H., Wang J., Jiang Y., Cheng Y., Lin P., Zhu H., Han G., Yi L., Zhang S., Guo L., Cheng S.: Typing of Canine Parvovirus Strains Circulating in North-East China. Transbound Emerg Dis 2017, 64, 495-503, doi: 10.1111/tbed.12390.

29. Zhao Y., Lin Y., Zeng X., Lu C., Hou J.: Genotyping and pathobiologic characterization of canine parvovirus circulating in Nanjing, China. Virol J 2013, 10, 272, doi: 10.1186/1743-422X10-272.

30. Zhao Z., Liu H., Ding K., Peng C., Xue Q., Yu Z., Xue Y.: Occurrence of canine parvovirus in dogs from Henan province of China in 2009-2014. BMC Vet Res 2016, 12, 138, doi: 10.1186/s12917-016-0753-1.

31. Zhou P., Zeng W., Zhang X., Li S.: The genetic evolution of canine parvovirus - A new perspective. PLoS One 2017, 12, e0175035, doi: 10.1371/journal.pone.0175035.

32. Zhuang L., Ji Y., Tian P., Wang K., Kou C., Gu N., Zhang Y.: Polymerase chain reaction combined with fluorescent lateral flow immunoassay based on magnetic purification for rapid detection of canine parvovirus 2. BMC Vet Res 2019, 15, 30, doi: 10.1186/s12917-019-1774-3. 